\title{
Balance between viscous and elastic parameters for ductile materials flow under an overdamped regime
}

Cite as: AIP Conference Proceedings 2116, 250004 (2019); https://doi.org/10.1063/1.5114244

Published Online: 24 July 2019

M. A. Forjaz, A. M. Almeida, T. de Lacerda-Arôso, and J. Pamplona

ARTICLES YOU MAY BE INTERESTED IN

Computer-aided optimization in additive manufacturing: Processing parameters and 3D scaffold reconstruction

AIP Conference Proceedings 2116, 230005 (2019); https://doi.org/10.1063/1.5114231

Envelope analysis of a habitable attic by the use of infrared thermography assessment AIP Conference Proceedings 2116, 250007 (2019); https://doi.org/10.1063/1.5114247

Micromechanical modelling of thermoelastic cracking in polycrystalline materials AIP Conference Proceedings 2116, 250002 (2019); https://doi.org/10.1063/1.5114242

Challenge us. What are your needs for periodic signal detection?

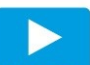

Watch

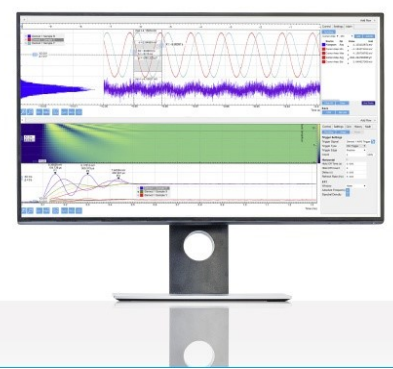

Zurich Instruments 


\title{
Balance Between Viscous and Elastic Parameters for Ductile Materials Flow under an Overdamped Regime
}

\author{
M. A. Forjaz ${ }^{1}$, A. M. Almeida ${ }^{2, \text { a) }}$, T. de Lacerda-Arôso ${ }^{2}$, J. Pamplona ${ }^{3, \text { b) }}$ \\ ${ }^{1}$ Centro de Matemática (CMAT), Departamento de Matemática e Aplicações, Universidade do Minho, Campus de \\ Gualtar, 4710-057 Braga, Portugal. \\ ${ }^{2}$ Centro de Física (CFUM), Departamento de Física, Universidade do Minho, Campus de Gualtar, 4710-057 \\ Braga, Portugal. \\ ${ }^{3}$ Instituto de Ciências da Terra (ICT) - Pólo da Universidade do Minho. Departamento de Ciências da Terra, \\ Universidade do Minho, Campus de Gualtar, 4710-057 Braga, Portugal. \\ a) Corresponding author: coimbra@fisica.uminho.pt \\ b) jopamp@dct.uminho.pt
}

\begin{abstract}
The evolution of less ductile layers embedded in a more ductile matrix under simple progressive shear in some geological structures, such as folding and asymmetric boudinage in the same direction, is a current trend in structural geology research. Aiming to study this subject, in this work a finite element method (FEM) is used to discretize a continuous medium as a point-mass model in which every node is subject to elastic and viscous forces. The dynamic equation of the present system takes into account both kinds of those forces driving the motion of each particle. In the herein developed model the system is subject to external shear forces applied to some nodes, resulting in a continuous flow of material. The balance between viscous and elastic constants is pursuit in order to characterize the evolution of the system.
\end{abstract}

\section{INTRODUCTION}

Geological processes in which materials with contrasting physical properties are in contact and subject to external stress generate interesting phenomena, namely folding and boudinage and the simultaneous occurrence of both of these structures have been attributed to different spatial directions [1]. Recently, a new interpretation proposes that they can occur in the same direction [2]. However, this trend still lacks a theoretical model, for which this work aims to be a contribution.

In the following sections we present the model developed, for which we show a brief study of a small system of interconnected mass-points, either without friction (which results in a system of coupled harmonic oscillators) or with friction between each pair of interconnected particles (within some range of viscosity). Finally, we point out some improvements that may be implemented in the model as well as application to systems with different ductile layers of materials.

\section{THE MODEL}

We propose a 2-D model to predict the evolution of a ductile material subject to external shear stress. A continuous mass is discretized in a finite set of mass-points interconnected within a predefined range. Thus, each pair of points, which lay apart further than the predefined range are not connected. 
A finite element method (FEM) is used to calculate ab initio the behavior of the whole system under the action of external forces and how it accommodates this strain.

The set of mass-points are distributed more or less regularly over the XY plane. An algorithm is applied in order to establish all the connections of each mass-point with all the others within the predefined range (e.g., only neighbor mass-points are connected), as it is shown in Fig. 1 when we consider, for example, 16 nodes.

This algorithm is to be applied whenever the program executes one step (by step we mean the application of an external force field), thus allowing the mass-points to reestablish or to cancel their connections according to the change of their positions relatively to their neighbors.

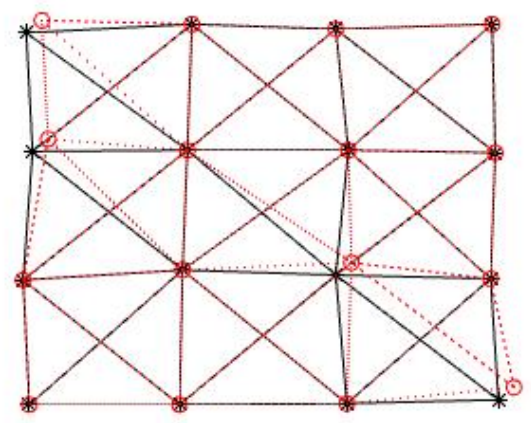

FIGURE 1. Each node of the truss stands for each mass-point and the lines represent the connection between every two points. In this picture it can be seen a starting relaxed geometry (asterisks connected by solid lines) and the new geometry after the first displacement imposed into 4 mass-points (circles connected by dotted lines).

Every pair of interconnected points is treated as an oscillator, and obeys Newton second law $F=m a$, where $F$ is the total force applied on mass $m$, which is subject to acceleration $a$.

Considering the starting geometry as a relaxed configuration of the system, we superimpose a first deformation corresponding to the external applied forces. Then, each oscillator will respond according to the physical parameters defined by the elasticity $(k)$ and the viscosity $(b)$ and, such that

$$
F=F_{\text {elastic }}+F_{\text {viscosity }}=-k u(t)-b \dot{u}(t)
$$

where $u(t)$ is the displacement, and $\dot{u}(t)$ is the velocity as a function of time $t$.

The balance between the elastic forces, governed by the elastic constant $k$, and the viscous forces, depending on the viscosity coefficient $b$, determines the regime of oscillation that can be observed for every mass-point. In real viscous materials flows the viscous forces opposing deformation overcome by large the elastic forces. Typically, the Young modulus for these materials ranges from $10^{7}$ to $10^{9} \mathrm{~N} / \mathrm{m}^{2}$ [3] and the friction coefficient is of the order of $10^{5}$ to $10^{9} \mathrm{~Pa}$.s [4]. Thus, the approach to these kinds of materials as a set of coupled oscillators is better described by considering it in an overdamping regime [5].

\section{CASE STUDY}

In this paper we present some tests made to the model concerning only the first step in order to verify its consistency and the convergence of the model considering it as a set of coupled oscillators. The ultimate test was to reduce the set of mass-points to only two, connected by a spring of stiffness $k$, either with or without damping driven by the viscosity coefficient $b$, related to the Stokes friction.

In the set of 16 mass-points depicted in Fig. 1 the average distance between neighboring points, both in $\mathrm{X}$ and $\mathrm{Y}$ directions, is slightly randomly distorted from a perfect square geometry. This is the relaxed starting geometry.

The application of a large displacement to some of the 16 mass-points, of the order of $1 \%$ relatively to the separation between mass-points (for the purpose of this study only), leads to oscillations of all points, as shown in Fig. 2 for one single mass. 


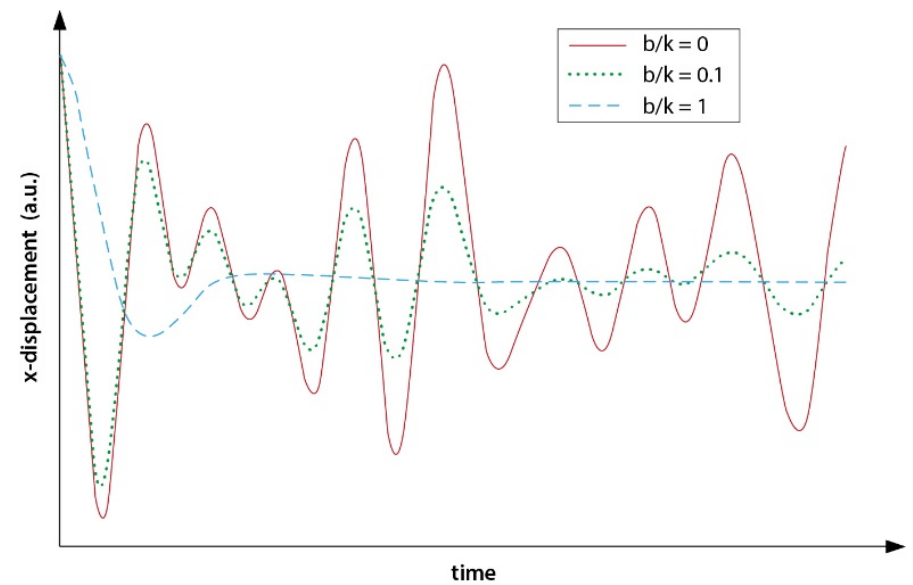

FIGURE 2. The displacement along the $X$ direction of one of the 16 mass-points as a function of time without friction (solid line); with moderate damping $b / k=0.1$ (dotted line); and for an overdamped regime when $b / k=1$ (dashed line).

In particular, the selected mass-point has: (i) a time-modulated oscillatory motion when there is no damping $(b=0)$ (solid line); (ii) a weak damping when $b / k=0.1$ (dotted line); (iii) a heavily damped movement for $b / k=1$ (dashed line). Similar results are obtained for every other particle in any direction X or Y.

In Fig. 3 it is shown the acceleration along the $\mathrm{X}$ direction for the same ratios $b / k$ of the same particle.

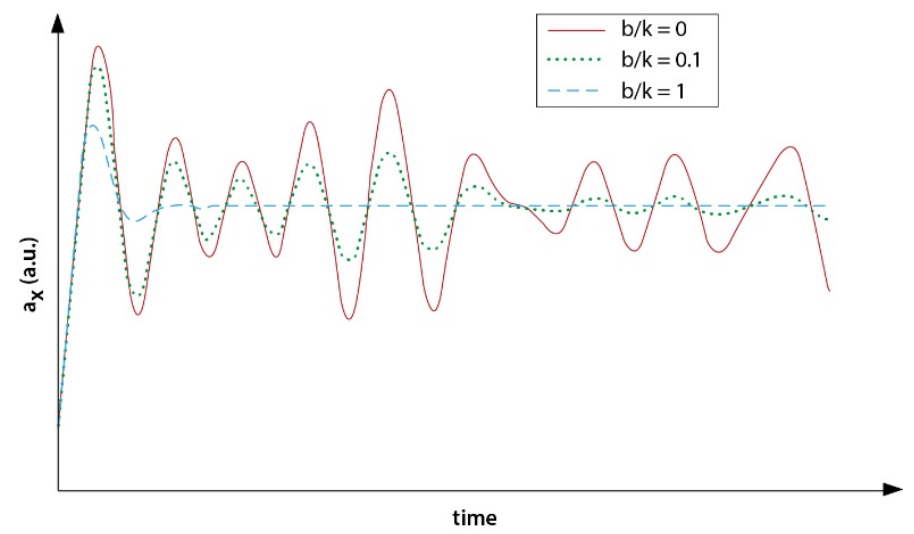

FIGURE 3. The acceleration along the $\mathrm{X}$ direction of one of the 16 mass-points as a function of time without friction (solid line); with moderate damping $b / k=0.1$ (dotted line); and for an overdamped regime when $b / k=1$ (dashed line).

The comparison of the plots in Fig. 2 and Fig. 3 also shows a phase difference of $\pi$ between position and acceleration, as is expected for a harmonic oscillator, even under moderate damping.

Reducing to a set of two mass-points, a pure oscillatory motion can be observed when $b=0$, which then turns to an exponential amplitude reduction, for a damping regime with $b / k=0.1$ (Fig. 4). 


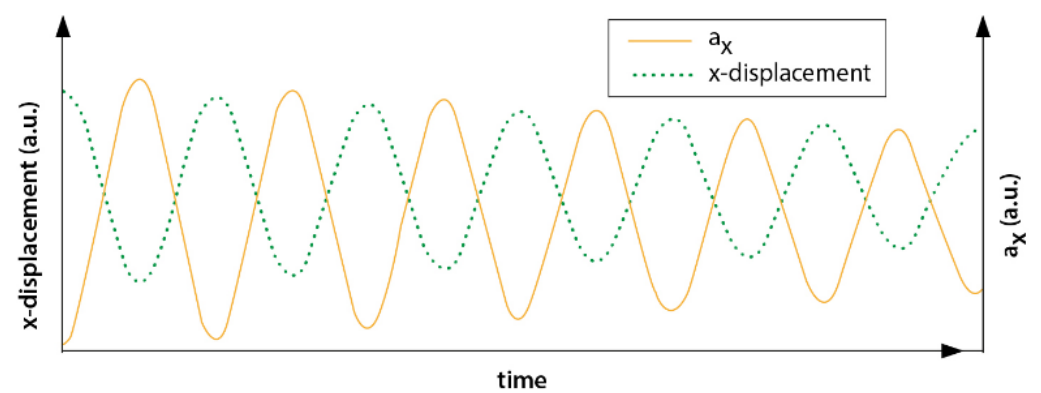

FIGURE 4. The displacement (dotted line) and the acceleration (solid line) along the $\mathrm{X}$ direction of one mass-point as a function of time for $b / k=0.1$.

\section{CONCLUSIONS AND FUTURE WORK}

The approach of a continuous mass system by a discrete set of point masses behaving as coupled oscillators seems to be reasonable and the reduction to a single pair of masses reproduces a harmonic oscillator. On the other hand, the extension to an arbitrarily large number of points is trivial.

The results presented are for an homogeneous material, but the model is conceived so to deal with different materials, e.g. different parameter such as volumic mass, elasticity and viscosity, thus allowing to build up layered structures.

This model has been working for only one step of external applied forces. The next evolution will make it work on a series of steps, for which the formulation as a Quadratic Eigenvalue Problem (QEP) $[6,7]$ is to be explored in order to implement a feasible, robust and time saving algorithm able to process a system of thousands of particles.

\section{ACKNOWLEDGMENTS}

The author Maria Antónia Forjaz acknowledges the funding supported by Portuguese Funds through FCT 'Fundação para a Ciência e a Tecnologia', UID/MAT/00013/2013. The author Jorge Pamplona is co-funded by the European Union through the European Regional Development Fund, based on COMPETE 2020 (Programa Operacional da Competitividade e Internacionalização), project ICT (UID/GEO/04683/2013) with reference POCI01-0145-FEDER-007690 and national funds provided by Fundação para a Ciência e Tecnologia. The authors Teresa de Lacerda-Arôso and António Mário Almeida acknowledge the support of the Portuguese Foundation for Science and Technology (FCT) in the framework of the Strategic Funding UID/FIS/04650/2013.

\section{REFERENCES}

[1] R. J. Twiss and E. M. Moores, Structural Geology (New Freeman \& Company, New York, 1992).

[2] J. Pamplona, B. C. Rodrigues and C. Fernández, Folding as a precursor of asymmetric boudinage in shear zones affecting migmatitic terranes, Geogaceta 55, 15-18 (2014).

[3] L. I. Vallejo, M. Ferrer, L. Ortuño and C. Oteo, Ingeniería Geológica (Pearson Educación, Madrid, 2005).

[4] M. E. Mengong and G. Zulauf, The impact of strain rate on folding and boudinage under plane strain: Results from analogue modeling, Geotectonic Research 95, 101-114 (2007).

[5] F. Tisseur and K. Meerbergen, The quadratic eigenvalue problem, SIAM Review 43 (2), 235-286 (2001).

[6] J. A. C. Martins, S. Barbarin, M. Raousan and A. Pinto da Costa, Dynamic stability of finite dimensional linearly elastic systems with unilateral contact and Coulomb friction, Computer Methods in Applied Mechanics and Engineering 177 (1), 289-328 (1999).

[7] M. A. Forjaz, A. M. Almeida, L. M. Fernandes, J. Pamplona and T. de Lacerda-Arôso, Approaching an overdamped system as a quadratic eigenvalue problem, Applied Mathematics \& Information Sciences 11 (4), 961-965 (2017). 